\title{
Cutaneous Mucormycosis Following a Bullous Pemphigoid Flare in a Chronic Lymphocytic Leukemia Patient on Ibrutinib
}

\author{
Matthew K. Stein ${ }^{\mathrm{a}, \mathrm{d}}$, Saradasri Karri ${ }^{\mathrm{b}}$, Jackson Reynolds ${ }^{\mathrm{b}}$, Jeff Owsley ${ }^{\mathrm{a}}$, Austin Wise ${ }^{\mathrm{b}}$, \\ Mike G. Martin ${ }^{\mathrm{a}, \mathrm{b}}$, Fereshteh Zare ${ }^{\mathrm{b}, \mathrm{c}}$
}

\begin{abstract}
While the recent development of novel therapeutics in oncology, such as small molecule kinase inhibitors (SMKIs), has enabled our ability to target disease-specific molecular pathways, the prolonged impact of these agents on the immune system and infectious risk remains to be seen. We present a 68-year-old male with refractory chronic lymphocytic leukemia (CLL) on ibrutinib monotherapy for 3 years who developed extensive cutaneous mucormycosis following a severe bullous pemphigoid (BP) flare. He received amphotericin B for 4 weeks and was continued on posaconazole with resolution of his mucormycosis infection. Consistent with a growing evidence of literature identifying opportunistic fungal infections in patients on ibrutinib therapy, providers should be cognizant of medical comorbidities that may predispose to such infections and explore methods of prevention before starting ibrutinib and other SMKIs.
\end{abstract}

Keywords: Ibrutinib; CLL; Mucormycosis; Small molecular kinase inhibitors; Fungal infection; Bullous pemphigoid

\section{Introduction}

Recent advances in oncology have led to the production of a number of precision therapies that affect molecular signaling pathways pertinent to a tumor's biology. While these new therapeutic targets, including small molecule kinase inhibitors

Manuscript submitted March 24, 2018, accepted April 4, 2018

${ }^{a}$ West Cancer Center and University of Tennessee Health Science Center, Memphis, TN, USA

${ }^{b}$ Department of Internal Medicine, University of Tennessee Health Science Center, Memphis, TN, USA

'Department of Palliative Medicine, Veterans Affairs Hospital, Memphis, TN, USA

${ }^{\mathrm{d} C}$ Corresponding Author: Matthew K. Stein, West Cancer Center and University of Tennessee Health Science Center, 7945 Wolf River Blvd, 3rd Floor Executive Suite, Germantown, Memphis, TN 38138, USA.

Email: mkstein@westclinic.com

doi: https://doi.org/10.14740/wjon1099w
(SMKI)s, are advantageous to conventional chemotherapy in their specificity and limitations on systemic toxicity, there is still much to learn regarding their long-term utility and side effect profile. In particular, the contribution of SMKIs on infectious risk in oncology patients already susceptible to immunosuppression remains to be elucidated. Here, we describe a patient with history of chronic lymphocytic leukemia (CLL) on ibrutinib therapy diagnosed with localized cutaneous mucormycosis following a significant bullous pemphigoid (BP) flare.

\section{Case Report}

A 68-year-old man presented to the hospital with a chief complaint of subjective fever, bilateral leg pain, worsening blistering and bleeding from BP lesions of the lower extremities. He had a chronic history of BP which was diagnosed over 4 years before and had a hospital admission 2 weeks prior for a severe flare of BP which necessitated treatment with prednisone 60 mg PO daily and a prolonged 6-week taper as well as doxycycline $100 \mathrm{mg}$ PO twice daily. A shave biopsy of the bullous skin lesions was completed by dermatology during the last admission and had not resulted at discharge. His other medical history was significant for refractory CLL as well as recurrent deep vein thrombosis on apixaban $5 \mathrm{mg}$ twice daily, chronic obstructive pulmonary disease, diastolic heart failure and noninsulin dependent diabetes mellitus. He was also being treated monthly for hypogammaglobulinemia with intravenous immunoglobulin $400 \mathrm{mg} / \mathrm{kg}$. Surgical history was unremarkable. He received the diagnosis of 11q deletion CLL in 1999 and previously underwent treatment with several regimens including rituximab, cyclophosphamide, vincristine and prednisone (R-CVP) as well as fludarabine and rituximab (FR). He was started on ibrutinib $420 \mathrm{mg}$ PO daily in 2014 for recurrence and was maintained on this medication for approximately 3 years before presentation to the hospital.

His initial vital signs were normal. Physical examination revealed multiple round hyperpigmented macules on the trunk, upper and proximal lower extremities. In addition, numerous necrotic ulcers with hemorrhagic keratotic eschars were interspersed with surrounding bullae from his recent BP flare. Comprehensive metabolic profile was within normal 


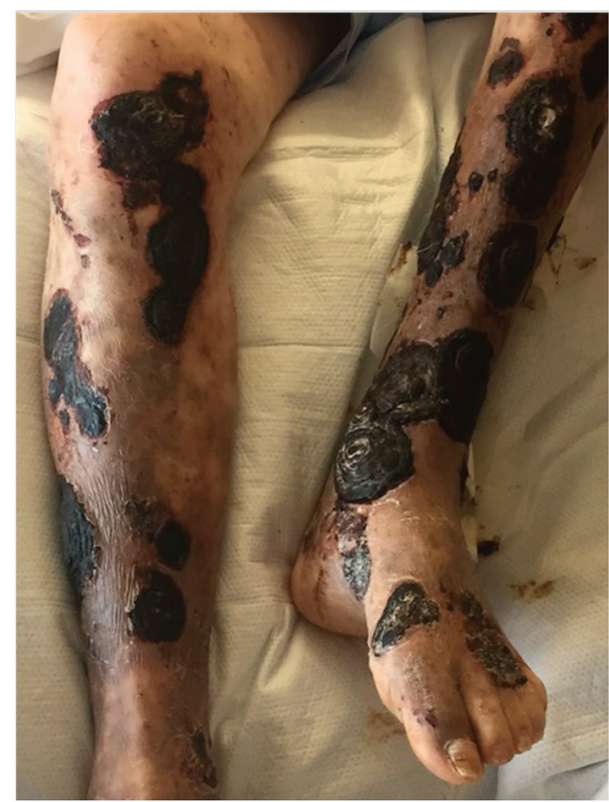

Figure 1. Photograph of CLL patient on ibrutinib with extensive necrosis and black eschars of lower extremity lesions following bullous pemphigoid flare.

limits. Complete blood count showed white blood cell count $2,600 / \mu \mathrm{L}$ (absolute neutrophil count $1,100 / \mu \mathrm{L}$ ), hemoglobin $9.3 \mathrm{~g} / \mathrm{dL}$ and platelets $103,000 / \mu \mathrm{L}$. He was given intravenous immunoglobulin as his quantitative immunoglobulins showed an $\mathrm{IgG}$ of $350 \mathrm{mg} / \mathrm{dL}$. Shortly after admission, the patient developed sepsis, atrial fibrillation with rapid ventricular response and hypotension and was subsequently transferred to the intensive care unit for central venous access and monitoring. He was started on broad spectrum antibiotics including vancomycin $15 \mathrm{mg} / \mathrm{kg}$ intravenous every $12 \mathrm{~h}$ and meropenem $1 \mathrm{~g}$ intravenous every $8 \mathrm{~h}$ per infectious disease recommendations. Ibrutinib was held, prophylactic acyclovir $400 \mathrm{mg}$ PO twice daily continued and prednisone for prior BP flare was tapered down to $20 \mathrm{mg}$ PO daily. Shave biopsy results of the lower extremity lesions from the previous hospitalization returned as trophozoites with concern for possible fungal infection and thus intravenous fluconazole $400 \mathrm{mg}$ daily was started.

The patient was stabilized in the intensive care unit over the course of several days and atrial fibrillation controlled with metoprolol. Vancomycin and meropenem were discontinued. Dermatology was consulted as new necrotic lesions continued to develop on his lower extremities despite fluconazole. Repeat punch biopsies were performed and the patient was switched to intravenous liposomal amphotericin B $5 \mathrm{mg} / \mathrm{kg}$ daily given concern for fungal etiology. Further workup which was negative included: blood cultures, cryptococcal antigen, urine histoplasma antigen, urine blastomycosis antigen and serum coccidioides antibody. Computed tomography of the chest, abdomen and pelvis with contrast was completed and significant for a hiatal hernia and splenomegaly; no other inflammatory or infectious process was identified on imaging.

He was transferred out of the intensive care unit and con- tinued on intravenous liposomal amphotericin B with appropriate wound care and pain control. An initial review of punch biopsies showed clusters of septate hyphae with chains of arthroconidia suggestive of fungal infection. X-rays of the bilateral tibia and fibula were unremarkable for bony abnormality and only showed subcutaneous skin thickening consistent with BP. On day 14 of amphotericin therapy, fungal cultures from punch biopsies returned positive for mucormycosis. By this time, there were extensive necrotic lesions with thick black eschars covering much of his lower extremities (Fig. 1). Several lesions appeared to be loosening and sloughing off. Bilateral magnetic resonance images of the tibia and fibula with and without contrast were negative for osteomyelitis; there was no evidence of extension of mucormycosis lesions beyond subcutaneous tissues. Plastic surgery was consulted for evaluation for debridement of his cutaneous mucormycosis to minimize deeper extension and dissemination; however, the decision was made to continue with antifungal therapy and aggressive wound care secondary to the extent of cutaneous disease and comorbidities.

On day 20 of amphotericin treatment, the patient developed foul smelling drainage with a green tinge at several lesions of the medial left lower extremity. His white blood cell count increased to $17,600 / \mu \mathrm{L}$ during this time. A wound culture was collected, and broad-spectrum antibiotics were initiated, including daptomycin intravenous $6 \mathrm{mg} / \mathrm{kg}$ daily and meropenem $1 \mathrm{~g}$ intravenous every $8 \mathrm{~h}$ for which he completed a 7-day course with improvement. Wound cultures grew Pseudomonas and E. coli which were both sensitive to ciprofloxacin. Repeat blood cultures were negative. The patient received 4 weeks of intravenous liposomal amphotericin B before he was transitioned to posaconazole $400 \mathrm{mg} \mathrm{PO}$ twice daily. His wounds continued to gradually heal and he was discharged to a skilled nursing facility after nearly 2 months of hospitalization.

\section{Discussion}

Mucormycosis is a general name given to a potentially lethal infection caused by filamentous fungi of the order Mucorales and is the third most common invasive fungus following Candida and Aspergillus [1]. While deemed relatively rare in the range of 1.7 cases per million in the United States [2], there is evidence that the incidence of mucormycosis is increasing $[3,4]$. The most common infectious sites for mucormycosis are the sinus and pulmonary systems, followed by cutaneous or gastrointestinal involvement $[3,5]$. Prompt diagnosis and treatment with liposomal amphotericin B and surgery is vital, as mortality rates are high, especially in patients with disseminated disease.

In the largest literature review of zygomycosis to date, Roden et al found the most common clinical characteristics of patients diagnosed with mucormycosis included: diabetes $(36 \%)$, malignancy $(17 \%)$, solid organ transplant $(7 \%)$, deferoxamine therapy $(6 \%)$, injection drug use $(5 \%)$, penetrating trauma (5\%), bone marrow transplantation (5\%) and surgery (3\%) [3]. Of patients with hematologic malignancies, mucormycosis most commonly occurred in patients with acute my- 
eloid or lymphoblastic leukemia, non-Hodgkin's lymphoma, hairy cell leukemia or multiple myeloma. Of two retrospective reviews, only $1 / 89$ patients with hematologic malignancies diagnosed with concomitant mucormycosis had CLL; none of the patients had primary cutaneous involvement as was seen in our case $[6,7]$.

Ibrutinib is a small-molecule, irreversible inhibitor of Bruton's tyrosine kinase (BTK), which normally serves as a downstream signal in the cytokine receptor and B-cell antigen receptor pathways. Indicated for use in cancers such as CLL/ small lymphocytic lymphoma (SLL), mantle cell lymphoma (MCL), Waldenstrom's macroglobulinemia, marginal zone lymphoma, and chronic graft versus host disease, inhibition of BTK with ibrutinib has been shown to limit malignant Bcell proliferation, cell migration, adhesion and survival in vivo [8]. According to the updated package insert, grade 3 or higher infections have occurred in $14-29 \%$ including bacterial, viral and fungal. Additionally, there was a significant risk of skin infections reported in trials of patients receiving ibrutinib for MCL (14\% all grades, 5\% grade 3-4) and CLL/SLL (16\% all grades, $6 \%$ grades $3-4$ ) [8].

Recent literature suggests that ibrutinib use may be associated with an increased risk for opportunistic fungal infections, including Cryptococcus neoformans, Pneumocystis jirovecii, and airborne filamentous fungi (e.g. Mucorales and Aspergillus) [9]. Chamilos et al identified 41 published hematologic malignancy patients (including 18 CLL cases) who developed invasive fungal infections while on ibrutinib therapy. In particular, the authors note that the atypical presentation of observed fungal infections including central nervous system and extrapulmonary disease not only led to increased mortality, but indicated that these patients could have had a complex immunodeficiency that extended beyond BTK-inhibition. They observe that invasive fungal infections in oncology patients appear to occur in those taking SMKIs which alter immune pathways such as PI3K, JAK/STAT and BTK signaling [10], and state the incidence reported during clinical trials is likely lower than in the general population as trial enrollment selected patients with fewer comorbidities impacting the immune system [9]. While the authors recognize there are a myriad of other predisposing factors which contribute to invasive fungal infections in patients taking ibrutinib and other SMKIs including cancer-induced immune defects [11], environmental exposure [12], genetic predisposition with polymorphisms in innate immune genes [13], and the effects of other immunosuppressive medications, it is vital to develop methods of early reporting of such infections during SMKI clinical trials and individualized approaches to preventing invasive fungal infections in those at high risk.

Consistent with our own PubMed search including keywords "CLL", "ibrutinib" and "mucormycosis", two other CLL cases with mucormycosis while on ibrutinib therapy have been published to our knowledge $[14,15]$. Of these, one site of infection was pulmonary and the other cutaneous; both of these patients died, although the patient with cutaneous mucormycosis was reported to have deceased secondary to unrelated causes after discontinuation of amphotericin B due to intolerance. In addition to his CLL and ibrutinib therapy, our patient had several comorbidities such as concomitant BP, recent ster- oid use and diabetes mellitus which all could have contributed to an immunodeficiency state leading to opportunistic infection. After 4 weeks of intravenous liposomal amphotericin B and maintenance posaconazole, his cutaneous mucormycosis lesions healed.

In conclusion, our case illustrates the importance of understanding the breadth of infectious risk in patients with hematological malignancies on ibrutinib and other SMKIs. Providers should be able to recognize patients with medical comorbidities putting them at an increased risk for invasive fungal infections including mucormycosis and perform a complete evaluation when the diagnosis is in question.

\section{Conflict of Interest}

The authors have no conflict of interest to disclose.

\section{References}

1. Petrikkos G, Skiada A, Lortholary O, Roilides E, Walsh TJ, Kontoyiannis DP. Epidemiology and clinical manifestations of mucormycosis. Clin Infect Dis. 2012;54(Suppl 1):S23-34.

2. Marr KA, Carter RA, Crippa F, Wald A, Corey L. Epidemiology and outcome of mould infections in hematopoietic stem cell transplant recipients. Clin Infect Dis. 2002;34(7):909-917.

3. Roden MM, Zaoutis TE, Buchanan WL, Knudsen TA, Sarkisova TA, Schaufele RL, Sein M, et al. Epidemiology and outcome of zygomycosis: a review of 929 reported cases. Clin Infect Dis. 2005;41(5):634-653.

4. Prabhu RM, Patel R. Mucormycosis and entomophthoramycosis: a review of the clinical manifestations, diagnosis and treatment. Clin Microbiol Infect. 2004;10(Suppl 1):31-47.

5. Lanternier F, Dannaoui E, Morizot G, Elie C, GarciaHermoso D, Huerre M, Bitar D, et al. A global analysis of mucormycosis in France: the RetroZygo Study (20052007). Clin Infect Dis. 2012;54(Suppl 1):S35-43.

6. Pagano L, Offidani M, Fianchi L, Nosari A, Candoni A, Picardi M, Corvatta L, et al. Mucormycosis in hematologic patients. Haematologica. 2004;89(2):207-214.

7. Noorifard M, Sekhavati E, Jalaei Khoo H, Hazraty I, Tabrizi R. Epidemiology and clinical manifestation of fungal infection related to Mucormycosis in hematologic malignancies. J Med Life. 2015;8(Spec Iss 2):32-37.

8. Ibrutinib (R) [package insert]. Horsham, PA USA: Janssen Biotech, Inc; 2017.

9. Chamilos G, Lionakis MS, Kontoyiannis DP. Call for action: invasive fungal infections associated with ibrutinib and other small molecule kinase inhibitors targeting immune signaling pathways. Clin Infect Dis. 2018;66(1):140-148.

10. Reinwald M, Boch T, Hofmann WK, Buchheidt D. risk of infectious complications in hemato-oncological patients treated with kinase inhibitors. Biomark Insights. 2015;10(Suppl 3):55-68. 
11. Forconi F, Moss P. Perturbation of the normal immune system in patients with CLL. Blood. 2015;126(5):573581 .

12. Bodey GP. Managing infections in the immunocompromised patient. Clin Infect Dis. 2005;40(Suppl 4):S239.

13. Cunha C, Aversa F, Bistoni G, Casagrande A, Rodrigues F, Romani L, Carvalho A. Immunogenetic profiling to predict risk of invasive fungal diseases: where are we now? Immunol Invest. 2011;40(7-8):723-734.

14. Kreiniz N, Bejar J, Polliack A, Tadmor T. Severe pneumonia associated with ibrutinib monotherapy for CLL and lymphoma. Hematol Oncol. 2018;36(1):349-354.

15. Figuera Castro C. Cutaneous mucormycosis in a patient with chronic lymphocytic leukemia: a pharmacological dilemma. Journal of Investigative Medicine. 2016;64:956-957. 